\title{
Acute respiratory failure and mechanical ventilation in cardiogenic shock complicating acute myocardial infarction in the USA, 2000- 2014
}

Saraschandra Vallabhajosyula ${ }^{1,2^{*}}$ (D), Kianoush Kashani ${ }^{2,3}$, Shannon M. Dunlay ${ }^{1,4}$, Shashaank Vallabhajosyula ${ }^{2}$, Saarwaani Vallabhajosyula ${ }^{1}$, Pranathi R. Sundaragiri ${ }^{5}$, Bernard J. Gersh ${ }^{1}$, Allan S. Jaffe ${ }^{1}$ and Gregory W. Barsness ${ }^{1}$

\begin{abstract}
Background: There are limited epidemiological data on acute respiratory failure (ARF) in cardiogenic shock complicating acute myocardial infarction (AMI-CS). This study sought to evaluate the prevalence and outcomes of ARF in AMI-CS.

Methods: This was a retrospective study of AMI-CS admissions during 2000-2014 from the National Inpatient Sample. Administrative codes for ARF and mechanical ventilation (MV) were used to define the cohorts of no ARF, ARF without MV and ARF with MV. Admissions with a secondary diagnosis of AMI and with chronic MV were excluded. Outcomes of interest included in-hospital mortality, temporal trends of ARF prevalence and resource utilization.
\end{abstract}

Measurements and main results: During 2000-2014, 439,436 admissions for AMI-CS met the inclusion criteria. ARF and $\mathrm{MV}$ were noted in $57 \%$ and $43 \%$, respectively. Admissions with non-ST-elevation AMI-CS, of non-White race and with non-private insurance received MV more frequently. Noninvasive ventilation and invasive MV increased from $0.4 \%$ and $39.2 \%$ (2000) to $3.6 \%$ and $46.4 \%$ (2014), respectively $(p<0.001)$. Coronary angiography and percutaneous coronary intervention were used less frequently in admissions receiving ARF with MV. Compared to admissions with no ARF, ARF without MV (adjusted odds ratio (aOR) 1.56 [95\% confidence interval (CI) 1.53-1.59]; $p<0.001$ ) and ARF with MV (aOR 2.50 [95\% Cl 2.47-2.54]; $p<0.001$ ) were associated with higher in-hospital mortality. Admissions with ARF without MV had greater resource utilization and lesser discharges to home as compared to no ARF.

Conclusions: In this contemporary AMI-CS cohort, the presence of ARF and MV use was noted in 57\% and 43\%, respectively, and was associated with higher in-hospital mortality.

Keywords: Cardiogenic shock, Acute myocardial infarction, Acute respiratory failure, Mechanical ventilation, Cardiac intensive care unit, Critical care cardiology, Outcomes research

\footnotetext{
*Correspondence: Vallabhajosyula.Saraschandra@mayo.edu

1 Department of Cardiovascular Medicine, Mayo Clinic, 200 First Street SW, Rochester, MN 55905, USA

Full list of author information is available at the end of the article
}

\section{SpringerOpen}

(c) The Author(s) 2019. This article is distributed under the terms of the Creative Commons Attribution 4.0 International License (http://creativecommons.org/licenses/by/4.0/), which permits unrestricted use, distribution, and reproduction in any medium, provided you give appropriate credit to the original author(s) and the source, provide a link to the Creative Commons license, and indicate if changes were made. 


\section{Introduction}

Cardiogenic shock (CS) is seen in about $5-7 \%$ of patients with acute myocardial infarction (AMI) and is associated with high mortality and morbidity [1-4]. In patients with AMI-CS, use of early revascularization may reverse the hemodynamic insult limiting pump failure and subsequent hemodynamic compromise [5, 6]. However, patients with AMI-CS can present with varying degrees of hemodynamic compromise, fluid overload and endorgan hypoperfusion [6-9]. In addition, these patients typically have high filling pressures, biventricular failure and secondary pulmonary hypertension resulting in decreased gas exchange and increased work of breathing, contributing to acute respiratory failure (ARF) [8]. Prior literature has demonstrated that noninvasive ventilation (NIV) reduces respiratory distress and improves metabolic disturbances in acute cardiogenic pulmonary edema [10]. However, in patients with CS, NIV might not always be feasible due to the high metabolic demand from increased work of breathing, altered mental status resulting in poor synchrony, concomitant cardiac arrest and severity of pulmonary edema with poor diuretic response causing insufficient oxygenation, all of which require tracheal intubation and the use of invasive mechanical ventilation (IMV) [11].

There are limited large-scale epidemiological data on the use of mechanical ventilation (MV) in the USA [12, 13]. Prior epidemiological studies have looked at the role of MV in medical intensive care unit (ICU) and cardiac ICU populations $[12,13]$. Recent data have noted increasing non-cardiac comorbidities in the cardiac ICU population, probably as a reflection of increasing severity of illness in this population [7, 13, 14]. Taking this background information into account, we sought to assess the epidemiology of ARF and MV in patients with AMI-CS in the USA. We hypothesized that during this 15-year study period, patients with AMI-CS have evolved into a more complex population with greater ARF and the use of MV, both NIV and IMV. We divided the population with AMI-CS into cohorts with no ARF, ARF without MV use and ARF with MV use.

\section{Materials and methods}

\section{Study population, variables and outcomes}

The National (Nationwide) Inpatient Sample (NIS) is the largest all-payer database of hospitalized inpatients in the USA and is a part of the Healthcare Cost and Utilization Project (HCUP), sponsored by the Agency for Healthcare Research and Quality [15]. During the study years, it contained data from about 1000 hospitals sampled to approximate a $20 \%$ sample of US community hospitals, defined by the American Hospital Association to be "all non-Federal, short-term, general and other specialty hospitals, excluding hospital units of institutions." The strata use five hospital characteristics: ownership/control, bed size, teaching status, urban/rural location and US region. The sample of hospitals included each year is independent of the sample included in preceding years. Information regarding each discharge includes demographics, primary payer, hospital characteristics, principal diagnosis, up to 24 secondary diagnoses and procedural diagnoses.

Using the HCUP-NIS data from 2000 to 2014, a retrospective cohort study of admissions with AMI-CS was identified. Though the Agency for Healthcare Research and Quality has released the HCUP-NIS data till 2016, due to the change in coding practices from ICD-9CM to ICD-10CM in October 2015 we sought to restrict the data to 2014. The HCUP-NIS from 2015 and 2016 databases lacks the Clinical Classification System for ICD-9CM codes used in the study. Furthermore, the ICD-10CM codes lack extensive validation studies unlike the ICD-9CM codes and therefore need further evaluation prior to incorporation into temporal analyses $[16,17]$. AMI in the primary procedure field was identified using International Classification of Diseases 9 Clinical Modification (ICD-9CM) codes for ST-elevation MI (STEMI) (ICD-9CM $410.1 \times-410.6 \times, 410.8 \times$, $410.9 \times)$ and non-ST-elevation acute coronary syndrome (NSTEMI) (ICD-9CM 410.70-410.79) [18]. CS was identified using ICD-9CM code 785.51 and was defined as shock resulting from diminution of cardiac output in heart disease, shock resulting from primary failure of the heart in its pumping function, as in myocardial infarction, severe cardiomyopathy or mechanical obstruction or compression of the heart or shock resulting from the failure of the heart to maintain adequate output [19]. Validation studies have shown a specificity of $99.3 \%$, a sensitivity of $59.8 \%$, a positive predictive value of $78.8 \%$ and negative predictive value of $98.1 \%$ for the ICD-9CM code 785.51 to identify CS [19]. Admissions with CS due to non-AMI etiology and those without in-hospital mortality data were excluded. Using previous algorithms applied to the HCUP-NIS database, ARF was identified using the presence of any of the following ICD-9CM codes: (a) acute respiratory failure (ICD-9CM 518.81), (b) other pulmonary insufficiency including acute respiratory distress syndrome and acute respiratory insufficiency (ICD-9CM 518.82), (c) acute respiratory distress syndrome after shock or trauma (ICD-9CM 518.85), (d) respiratory distress not otherwise specified (ICD-9CM 786.09), (e) respiratory arrest (799.1) and (f) ventilator management (ICD-9CM 96.7, 96.70, 96.71 and 96.72) [7, 20-22]. Use of MV was identified using ICD-9CM codes for NIV (ICD-9CM 93.90) and IMV (ICD-9CM 96.7, 96.70, 96.71, 96.72) [12]. The ICD-9CM for NIV and IMV 
is $86 \%$ sensitive $/ 92 \%$ specific and $86 \%$ sensitive/99.7\% specific, respectively [23]. Since it is possible that NIV might have been used for other purposes outside of ARF (obstructive sleep apnea, sleep disorder breathing), we excluded admissions when NIV was used without a concomitant diagnosis of ARF. Demographic and hospital characteristics associated with each discharge were identified from the HCUP-NIS database. Prior validated methodology was used to define acute organ dysfunction, cardiac and non-cardiac procedures [7, 22, 24-29]. The Deyo's modification of the Charlson comorbidity index was used to identify the burden of comorbid diseases (Additional file 1: Table S1) [30]. The hospital day on which the procedure was performed was used to identify the use of NIV before or after IMV in admissions that received both modalities.

The primary outcome was the in-hospital mortality in AMI-CS stratified into cohorts with no ARF, ARF without MV and ARF with MV. Secondary outcomes included the prevalence, temporal trends of ARF, length of stay, costs, use of do-not-resuscitate status and discharge disposition in admissions with ARF with/without MV.

\section{Statistical analysis}

As recommended by HCUP-NIS, survey procedures using discharge weights provided with HCUP-NIS database were used to generate national estimates. As recommended by HCUP-NIS, survey procedures using discharge weights provided with HCUP-NIS database were used to generate national estimates. Using the trend weights provided by the HCUP-NIS, samples from 2000 to 2011 were re-weighted to adjust for the 2012 HCUP-NIS re-design [31]. In 2012, the HCUP-NIS was re-designed to sample $20 \%$ of the national patient-level sample as compared to 2000-2011 wherein it sampled $100 \%$ of the discharges from $20 \%$ of the hospitals [31]. Using trend weights available on the HCUP-NIS database, samples from 2000 to 2011 were retroactively reweighted. The new sampling strategy is expected to result in more precise estimates than the previous HCUP-NIS design by reducing sampling error [15]. This methodology has been used by multiple prior studies spanning across year 2012 from the HCUP-NIS [7, 22, 24-29]. One-way analysis of variance (ANOVA) and $t$-tests were used to compare categorical and continuous variables, respectively. The inherent restrictions of the HCUP-NIS database related to research design, data interpretation and data analysis were reviewed and addressed [31]. Univariate analysis for trends of ARF and in-hospital mortality stratified by ARF with/without MV was represented as odds ratio (OR) with $95 \%$ confidence interval (CI). For the adjusted analysis, a multivariable logistic regression analysis including age, sex, race, admission year, primary payer status, socioeconomic stratum, hospital characteristics, comorbidities, acute organ dysfunction, severe sepsis, cardiac arrest, cardiac procedures, mechanical circulatory support and hemodialysis was performed for in-hospital mortality. For the multivariable modeling, purposeful selection of statistically $(p<0.20)$ and a priori selected clinically relevant variables was conducted. Additionally, we performed a propensity-matched analysis for demographics, comorbidities, hospital characteristics, acute organ failure and acute care interventions between the two cohorts. For the propensity matching, all variables except race had $<1 \%$ missing variables. For the race category, missing variables were imputed using random sampling from the respective covariate distributions. Using 1:1 nearest-neighbor matching, 9240 matching pairs $(18,480$ individual admissions) were developed for further use. The propensity-matched sample had standardized differences $<10 \%$ for all baseline characteristics. The McNemar $\chi^{2}$ test and paired sample $t$-tests were used to compare categorical and continuous variables, respectively, in the propensity-matched sample. Two-tailed $p<0.05$ was considered statistically significant. All statistical analyses were performed using SPSS version 25.0 (IBM Corp, Armonk, NY).

\section{Results}

There were an estimated number of 444,253 admissions for AMI-CS between January 1, 2000, and December 31, 2014, that met criteria for a primary diagnosis of STEMI or NSTEMI. An estimated number of 4817 (1.1\%) admissions received NIV without a concomitant diagnosis of ARF and were excluded. In the final cohort of 439,436 admissions, ARF was noted in 247,898 (56.5\%) with use of MV in 189,848 (43.2\%). In these 189,848 admissions, NIV was used in 8895 (4.7\%), IMV in 185,589 (97.8\%) and both in 4636 (2.4\%). Baseline characteristics of the cohorts with no ARF, ARF without MV and ARF with MV are summarized in Table 1. MV was used more frequently among admissions with NSTEMI-CS, of nonWhite race and with non-private insurance and to urban teaching hospitals. Over the 15-year study period, there was a steady decline in AMI-CS with no ARF with a concomitant increase in AMI-CS with ARF needing MV (Fig. 1a, b). Epidemiological trends of ARF and MV stratified by demographic and hospital characteristics are presented in Additional file 2: Figure S1 and Additional file 3: Figure S2. The timing of NIV with respect to IMV was available in 3866/4636 (83.4\%) admissions (Fig. 2). Nearly one-third admissions received NIV and IMV on the same day (Fig. 2). Admissions with ARF needing MV had higher rates of concomitant cardiac arrest, acute kidney injury and invasive hemodynamic assessment (Table 1). Coronary angiography and percutaneous 
Table 1 Baseline and hospital characteristics of AMI-CS with and without ARF

\begin{tabular}{|c|c|c|c|c|}
\hline Characteristic & No ARF $(N=191,538)$ & $\begin{array}{l}\text { ARF without MV } \\
(N=58,050)\end{array}$ & $\begin{array}{l}\text { ARF with MV } \\
(N=189,848)\end{array}$ & $p$ \\
\hline \multicolumn{5}{|l|}{ AMI type } \\
\hline STEMI & 69.8 & 69.1 & 66.5 & $<0.001$ \\
\hline NSTEMI & 30.2 & 30.9 & 33.5 & $<0.001$ \\
\hline Age (years) & $69.5 \pm 13.6$ & $68.7 \pm 12.8$ & $69.1 \pm 13.1$ & $<0.001$ \\
\hline Female sex & 40.3 & 38.5 & 38.2 & $<0.001$ \\
\hline \multicolumn{5}{|l|}{ Race } \\
\hline White & 63.1 & 60.0 & 63.9 & \multirow[t]{2}{*}{$<0.001$} \\
\hline Non-White & 36.9 & 40.0 & 36.1 & \\
\hline Weekend admission & 26.3 & 27.4 & 27.4 & $<0.001$ \\
\hline \multicolumn{5}{|l|}{ Primary payer } \\
\hline Medicare & 61.5 & 60.3 & 61.7 & \multirow[t]{3}{*}{$<0.001$} \\
\hline Medicaid & 5.5 & 6.3 & 7.2 & \\
\hline Others & 33.0 & 33.4 & 31.1 & \\
\hline \multicolumn{5}{|c|}{ Quartile of median household income for zip code } \\
\hline $0-25$ th & 22.8 & 24.7 & 23.2 & \multirow[t]{4}{*}{$<0.001$} \\
\hline 26th-50th & 26.8 & 27.0 & 26.2 & \\
\hline 51st-75th & 25.2 & 24.3 & 25.0 & \\
\hline 75th-100th & 25.2 & 24.0 & 25.6 & \\
\hline \multicolumn{5}{|l|}{ Hospital teaching status and location } \\
\hline Rural & 9.6 & 6.3 & 5.7 & \multirow[t]{3}{*}{$<0.001$} \\
\hline Urban non-teaching & 41.4 & 40.1 & 40.2 & \\
\hline Urban teaching & 49.0 & 53.6 & 54.2 & \\
\hline \multicolumn{5}{|l|}{ Hospital bed size } \\
\hline Small & 8.3 & 7.4 & 7.3 & \multirow[t]{3}{*}{$<0.001$} \\
\hline Medium & 22.3 & 21.6 & 22.1 & \\
\hline Large & 69.4 & 71.0 & 70.6 & \\
\hline \multicolumn{5}{|l|}{ Hospital region } \\
\hline Northeast & 18.3 & 15.2 & 19.6 & \multirow[t]{4}{*}{$<0.001$} \\
\hline Midwest & 23.3 & 23.1 & 22.5 & \\
\hline South & 38.8 & 44.9 & 35.9 & \\
\hline West & 19.5 & 16.8 & 22.0 & \\
\hline \multicolumn{5}{|l|}{ Charlson comorbidity index } \\
\hline $0-3$ & 27.0 & 23.6 & 22.0 & \multirow[t]{3}{*}{$<0.001$} \\
\hline $4-6$ & 53.3 & 57.4 & 57.2 & \\
\hline$\geq 7$ & 19.7 & 19.0 & 20.8 & \\
\hline \multicolumn{5}{|l|}{ Comorbidities } \\
\hline Hypertension & 51.5 & 42.7 & 51.0 & $<0.001$ \\
\hline Hyperlipidemia & 35.9 & 25.7 & 29.1 & $<0.001$ \\
\hline Diabetes mellitus & 4.0 & 4.5 & 4.7 & $<0.001$ \\
\hline Cancer & 7.5 & 5.1 & 6.6 & $<0.001$ \\
\hline CKD & 12.1 & 13.5 & 14.5 & $<0.001$ \\
\hline Heart failure & 49.6 & 62.6 & 59.0 & $<0.001$ \\
\hline Cardiac arrest & 8.2 & 18.6 & 28.0 & $<0.001$ \\
\hline Acute kidney injury & 24.7 & 40.9 & 43.7 & $<0.001$ \\
\hline Coronary angiography & 68.6 & 75.7 & 64.9 & $<0.001$ \\
\hline Percutaneous coronary intervention & 48.8 & 56.4 & 43.4 & $<0.001$ \\
\hline Invasive hemodynamic assessment ${ }^{a}$ & 15.6 & 22.2 & 23.7 & $<0.001$ \\
\hline Severe sepsis & 2.6 & 6.7 & 9.3 & $<0.001$ \\
\hline
\end{tabular}


Table 1 (continued)

\begin{tabular}{|c|c|c|c|c|}
\hline Characteristic & No ARF $(N=191,538)$ & $\begin{array}{l}\text { ARF without MV } \\
(N=58,050)\end{array}$ & $\begin{array}{l}\text { ARF with MV } \\
(N=189,848)\end{array}$ & $p$ \\
\hline \multicolumn{5}{|l|}{ Cardiac surgery } \\
\hline CABG & 19.3 & 21.7 & 13.9 & $<0.001$ \\
\hline Valve surgery & 1.6 & 3.2 & 1.9 & $<0.001$ \\
\hline \multicolumn{5}{|l|}{ MCS } \\
\hline Total & 41.5 & 56.7 & 45.5 & $<0.001$ \\
\hline IABP & 40.6 & 54.6 & 44.0 & $<0.001$ \\
\hline pMCS & 0.8 & 2.2 & 1.7 & $<0.001$ \\
\hline npMCS & 0.4 & 0.9 & 0.5 & $<0.001$ \\
\hline ECMO & 0.3 & 1.2 & 0.6 & $<0.001$ \\
\hline Hemodialysis & 1.5 & 2.8 & 5.7 & $<0.001$ \\
\hline
\end{tabular}

Represented as percentage or mean \pm standard deviation

$A M I$ acute myocardial infarction, $A R F$ acute respiratory failure, $C A B G$ coronary artery bypass grafting, $C K D$ chronic kidney disease, $C S$ cardiogenic shock, $E C M O$ extracorporeal membrane oxygenation, IABP intra-aortic balloon pump, MCS mechanical circulatory support, $M V$ mechanical ventilation, $n p M C S$ non-percutaneous mechanical circulatory support, NSTEMI non-ST-elevation myocardial infarction, pMCS percutaneous mechanical circulatory support, STEMI ST-elevation myocardial infarction

${ }^{a}$ Right heart catheterization or pulmonary artery catheterization; all comparisons made using one-way analysis of variance

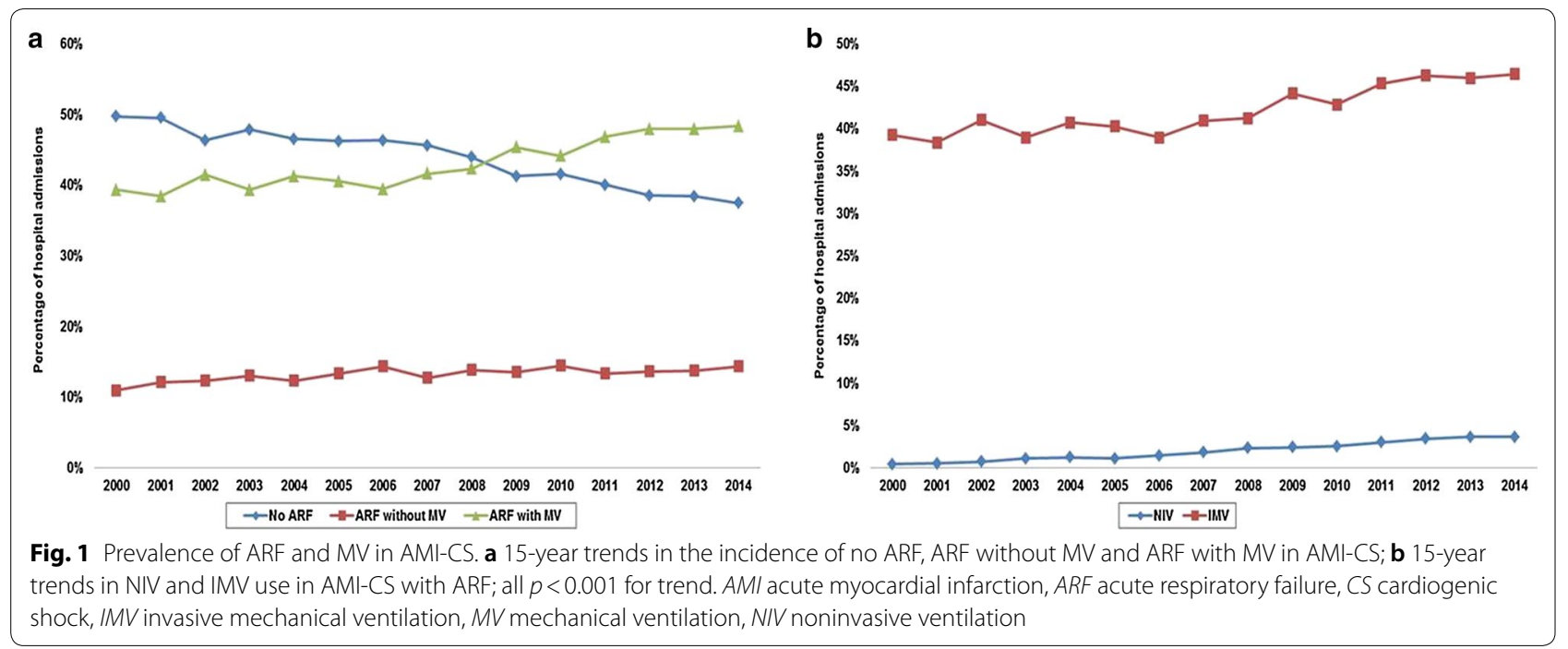

coronary intervention were used less frequently in ARF with MV (Table 1). Admissions with ARF without MV received mechanical circulatory support more frequently compared to those with no ARF; however, those with ARF with MV received it less frequently compared to ARF without MV.

Compared to those with no ARF, ARF without MV (37.8\% vs. 28.1\%; OR 1.56 [95\% CI 1.53-1.59]; $p<0.001$ ) and ARF with MV (49.4\% vs. $28.1 \%$; OR 2.50 [95\% CI 2.47-2.54]; $p<0.001)$ were associated with higher inhospital mortality. In-hospital mortality for the overall population and the ARF cohorts demonstrated a steady decline over the study period (Fig. 3). Admissions with
ARF without MV had a longer length of stay, higher hospital costs and lesser discharges to home as compared to those without ARF (Table 2). Admissions with ARF with MV had lower hospital costs and lengths of stay compared to ARF without MV but had greater use of donot-resuscitate status ( $5.6 \%$ vs. $4.5 \% ; p<0.001)$. In a multivariate regression analysis, compared to the cohort with no ARF, ARF without MV (OR 1.68 [95\% CI 1.64-1.72]; $p<0.001$ ) and ARF with MV (OR 2.21 [95\% CI 2.17-2.25]; $p<0.001)$ were independently associated with higher inhospital mortality in AMI-CS (Additional file 1: Table S2) (c-index 0.80; Hosmer and Lemeshow test for goodness of fit: $\left.\chi^{2} 1019 ; p<0.001\right)$. In a propensity-matched 


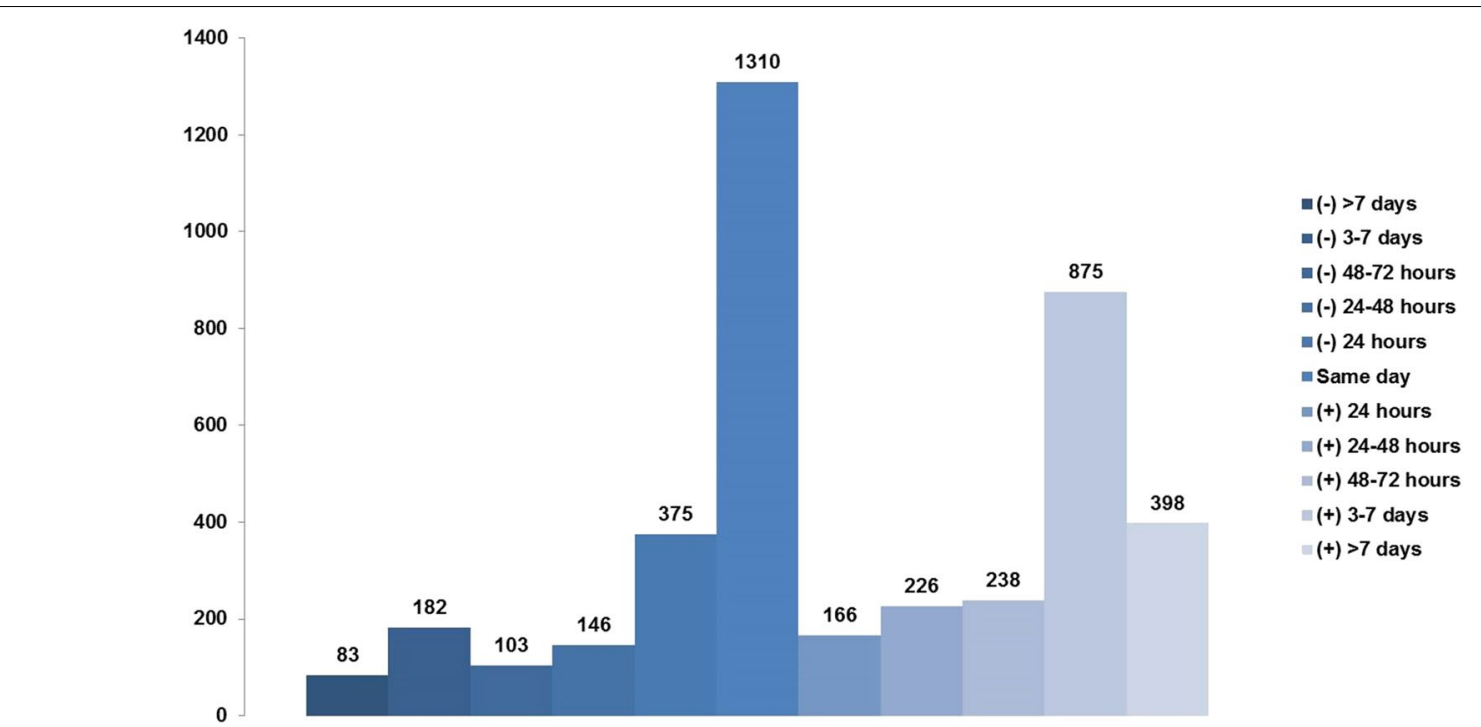

Fig. 2 Timing of NIV with relation to IMV (N=3866). Negative values denote NIV use before IMV and positive values denote NIV use after IMV. IMV invasive mechanical ventilation, NIV noninvasive ventilation

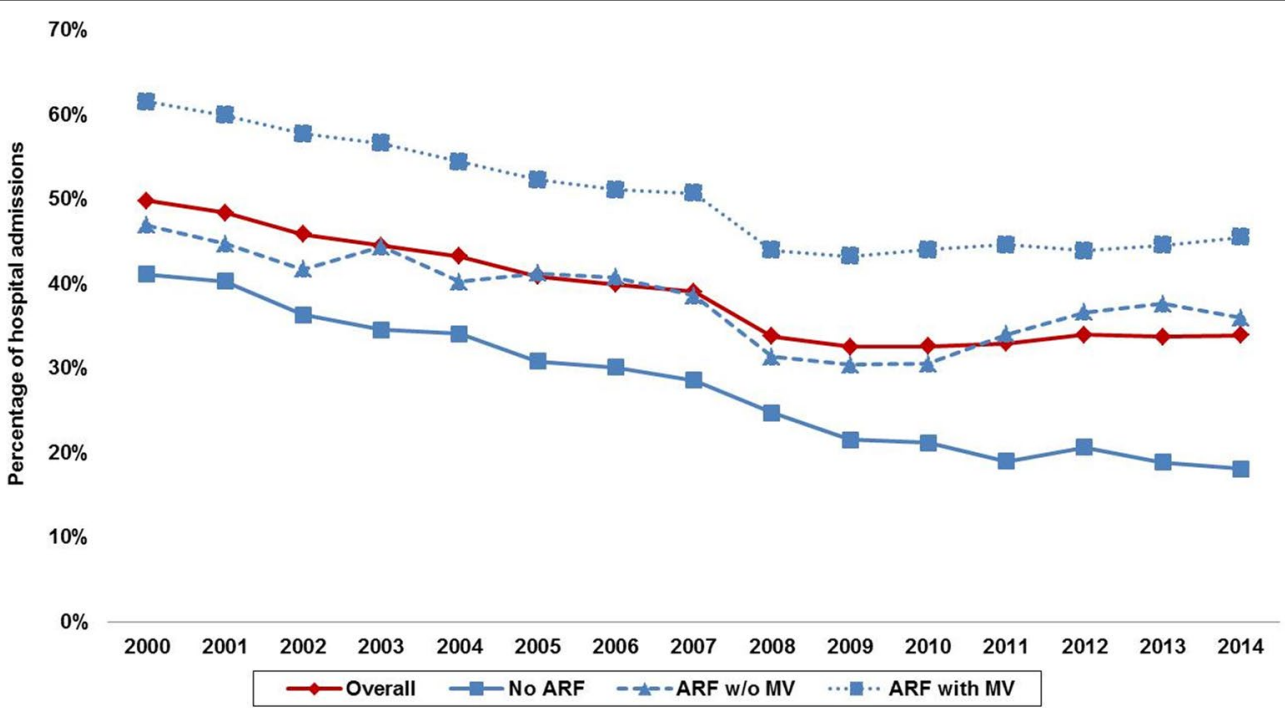

Fig. 3 Trends of in-hospital mortality in AMI-CS stratified by ARF and MV. All $p<0.001$. AMl acute myocardial infarction, ARF acute respiratory failure, CS cardiogenic shock, MV mechanical ventilation

analysis (Additional file 1: Table S3), compared to those without MV, the cohort receiving MV (38\% vs. $44.2 \%$; OR 1.29 [95\% CI 1.22-1.37]; $p<0.001$ ) continued to demonstrate higher in-hospital mortality.

\section{Discussion}

In this nationally representative population of AMICS, we noted a steady increase in the proportion of admissions with ARF and greater use of NIV and IMV between 2000 and 2014. Acute respiratory failure was seen more commonly in admissions with NSTEMICS, of non-White race and of male sex. The population with ARF with MV was less likely to receive coronary angiography and percutaneous coronary intervention. Acute respiratory failure without and with MV was associated with a 1.7- and 2.2-fold higher in-hospital mortality independent of baseline characteristics, the 
Table 2 Clinical outcomes of AMI-CS with and without ARF

\begin{tabular}{|c|c|c|c|c|}
\hline Characteristic & No ARF $(N=191,538)$ & ARF without MV $(N=58,050)$ & ARF with MV $(N=189,848)$ & $p$ \\
\hline In-hospital mortality & 28.1 & 37.8 & 49.4 & $<0.001$ \\
\hline Median length of stay (days) & $8.1 \pm 8.9$ & $12.4 \pm 12.6$ & $11.5 \pm 13.4$ & $<0.001$ \\
\hline Median hospitalization costs ( $\times 1000$ USD) & $95 \pm 115$ & $159 \pm 181$ & $148 \pm 176$ & $<0.001$ \\
\hline Do-not-resuscitate status & 3.0 & 4.5 & 5.6 & $<0.001$ \\
\hline \multicolumn{5}{|l|}{ Discharge disposition } \\
\hline Home & 37.3 & 23.2 & 15.7 & $<0.001$ \\
\hline Transfer & 6.9 & 5.6 & 7.6 & \\
\hline SNF & 15.7 & 21.9 & 18.6 & \\
\hline Home with $\mathrm{HHC}$ & 11.7 & 11.1 & 8.3 & \\
\hline AMA & 0.3 & 0.2 & 0.3 & \\
\hline
\end{tabular}

Represented as percentage or mean \pm standard deviation; all comparisons made using one-way analysis of variance

$A M A$ against medical advice, $A M I$ acute myocardial infarction, $A R F$ acute respiratory failure, $C S$ cardiogenic shock, $H H C$ home health care, $M V$ mechanical ventilation, SNF skilled nursing facility, USD US dollars

severity of illness and organ support. Similar findings were noted in the propensity-matched cohort.

\section{Epidemiology of acute respiratory failure in AMI-CS}

Acute respiratory failure requiring $\mathrm{MV}$ continues to be a leading reason for admission to the ICU. In unselected critically ill patients, Mehta et al. [12] noted a steady increase in the use of IMV in the USA between 1993 and 2009. In this study, the subgroup with heart failure (without CS) was noted to have a relatively steady usage of IMV during the study period. Using a registry of 219 patients, Hongisto et al. [11] described the use of NIV and IMV in unselected CS. They noted a $12 \%$ overall incidence of NIV use and 63\% IMV use during the 2-year study period. In contrast to these studies, our findings demonstrate an increasing incidence of ARF requiring MV. Furthermore, the use of NIV was noted in only $4.7 \%$ of our study population as compared to $12 \%$ in the CardShock trial [11]. In our study, the use of MV was noted in $43.2 \%$ of the population, which was significantly lower than the CardShock and IABP-SHOCK II (Intra-aortic Balloon Pump in Cardiogenic Shock II) cohorts [11, 32]. This can possibly be explained by the vast heterogeneity in the definition of CS employed in real-world registry data as compared to trial definitions. Additionally, differences in patient acuity and treatment between the USA and European populations may contribute to these differences. Our findings are consistent with data from other epidemiological studies that show greater use of IMV in male patients, non-White race and lower socioeconomic status [12]. In a population of 3.2 million noncardiogenic ARF, Cooke et al. noted consistently higher rates of ARF in non-White patients. The reasons for these disparities are incompletely understood and may be due to decreased access to health care, late presentation, differences in cultural and religious beliefs and treatment preferences. Further quantitative research investigating these racial disparities is warranted.

Expectedly, we noted ARF to be associated with higher occurrence of end-organ failure and cardiac arrest. In patients with CS, biventricular dysfunction may result in complex hemodynamics in the setting of positive pressure MV [33, 34]. Since the HCUP-NIS database does not record hemodynamic or echocardiographic data, we could not discern bi- from single-ventricular failure among participants. Furthermore, patients with AMI-CS frequently develop acute metabolic acidosis and vasoplegic shock [35], so it is conceivable that they develop a capillary leak syndrome or acute respiratory distress syndrome from concomitant sepsis resulting in worsening ARF. The increasing rates of MV in this study is consistent with prior studies that note greater acuity of patients being admitted to cardiac ICUs [13, 14]. This has significant implications on the models of care and staffing in modern cardiac ICUs, which include but are not limited to, dual-trained cardiac intensivists, co-management of patients by cardiologists and intensivists and development of specialized nursing care covering aspects unique to both cardiac and medical ICU populations [36].

\section{Mortality with acute respiratory failure in AMI-CS}

Multiorgan failure has been recognized as a significant contributor to morbidity and mortality in unselected medical and cardiac ICU patients [14, 37]. Recent AMI-CS prognostic scores have sought to incorporate measures of end-organ hypoperfusion into the risk stratification of these patients [7, 38, 39]. Consistent with these data, our study highlighted the incremental in-hospital mortality in admissions with ARF and with MV use. It is important to note that patients with ARF and MV 
received lesser mechanical circulatory support and had higher mortality and lesser utilization. Taken in aggregate, these data may suggest that use of MV was a marker of higher illness severity and therefore these patients died earlier during their hospital course. Our data can be readily compared to the subgroup of patients with CS $(n=600)$ enrolled in the Acute Heart Failure Database (AHEAD) registry [40]. In this study of unselected CS patients, the use of NIV, IMV or both was associated with $69 \%, 72 \%$ and $68 \%$ mortality, respectively, which was significantly higher than patients not receiving MV (i.e., 40\%) [40]. The mortality rate for the cohort with ARF and MV was only $40 \%$ in our study, which is similar to the data from the CardShock registry [11]. These observed differences are likely due to multiple factors: (a) the CardShock population had a higher proportion of AMI-CS as compared to the AHEAD registry and patients who develop post-cardiotomy CS appear to be systematically different from the AMI-CS [8]; (b) the AHEAD registry represented a referral population to centers of excellence in Europe, which cannot be generalized to our study that is more representative of the national practice; and (c) there were significant differences in the use of specific vasoactive medications (such as levosimendan) that are not available in the USA, thereby preventing direct comparisons. In 219 patients with AMI-CS, Hongisto et al. did not note the MV strategy (NIV or IMV) to impact clinical outcomes in their population. Further dedicated studies are needed to understand the role, indications and contraindications to NIV in CS [8].

\section{Limitations}

This study has several limitations, despite the HCUPNIS database's attempts to mitigate potential errors by using internal and external quality control measures. The ICD-9CM codes for AMI and CS have been previously validated that reduces the inherent errors in the study $[18,19]$. Important factors such as the timing of ARF, the presence of ARF at admission and treatmentlimiting decisions of organ support could not be reliably identified in this database. It is possible that there may be a hesitancy to intubate older or complicated patients that is reflected in the lower use of MV in this population. Importantly, change in respiratory function during the hospital stay (improvement or decline) could not be reliably assessed in all admissions, though an indirect assessment was available in admissions with a listed procedure day for NIV and IMV use. It is possible that despite best attempts at controlling for confounders by multivariate analysis, the use of MV is a marker of greater illness severity due to residual confounding. Echocardiographic data, mechanical ventilation data, sedation and paralysis, vasoactive medications and hemodynamic parameters were unavailable in this database. It is possible that sensitive definitions of ARF and the use of $\mathrm{MV}$ at lower thresholds of acuity may contribute to the increase in the prevalence of ARF and MV. However, the concomitant rise in other organ failure refutes this possibility. Despite these limitations, this study addresses an important knowledge gap highlighting the epidemiology of ARF and the use of MV in AMI-CS in a contemporary 15-year period.

\section{Conclusions}

In this study of 439,436 admissions with AMI-CS, ARF affected nearly $57 \%$ of the total cohort with a significant increase in the use of MV over time. Acute respiratory failure with or without MV use was independently associated with higher in-hospital mortality. Further research is needed to understand the delicate cardiopulmonary interactions in AMI-CS with an emphasis on ways to prevent and limit the severity of concomitant ARF.

\section{Supplementary information}

Supplementary information accompanies this paper at https://doi. org/10.1186/s13613-019-0571-2.

Additional file 1: Table S1. Administrative codes used for identification of diagnoses and procedures. Table S2. Multivariable regression for in-hospital mortality in AMI-CS. Table S3. Baseline characteristics of propensity-matched cohorts of AMI-CS.

Additional file 2: Figure S1. Trends of ARF and MV in AMI-CS stratified by demographic characteristics. Fifteen-year trends in acute respiratory failure (solid line) and mechanical ventilation (dashed line) in admission stratified by age groups (2A), race (2B), sex (2C) and Charlson comorbidity index groups (2D); all $p<0.001$. AMl: acute myocardial infarction; ARF: acute respiratory failure; CS: cardiogenic shock; $\mathrm{MV}$ : mechanical ventilation.

Additional file 3: Figure S2. Trends of ARF and MV in AMI-CS stratified by hospital characteristics. Fifteen-year trends in acute respiratory failure (solid line) and mechanical ventilation (dashed line) in admission stratified by hospital location and teaching status (3A), hospital bed size (3B) and hospital region (3C); all $p<0.001$. AMl: acute myocardial infarction; ARF: acute respiratory failure; CS: cardiogenic shock; MV: mechanical ventilation.

\section{Abbreviations}

AMI: acute myocardial infarction; ARF: acute respiratory failure; $\mathrm{Cl}$ : confidence interval; CS: cardiogenic shock; HCUP: Healthcare Cost and Utilization Project; ICD-9CM: International Classification of Diseases-9 Clinical Modification; ICU: intensive care unit; IMV: invasive mechanical ventilation; MV: mechanical ventilation; NIS: National Inpatient Sample; NIV: noninvasive ventilation; NSTEMI: non-ST-elevation myocardial infarction; OR: odds ratio; STEMI: ST-elevation myocardial infarction.

\section{Acknowledgements}

None.

\section{Prior presentation}

Slide Presentation, 48th Critical Care Congress, Society of Critical Care Medicine, San Diego, California (February 2019). 


\section{Authors' contributions}

SarV, KK, SMD, ShaV and SaaV were involved in study design, literature review, data analysis and statistical analysis. SarV, ShaV and SaaV were involved in data management, analyzed the data and drafted the manuscript. SarV, KK, SMD, ShaV, SaaV, BJG, ASJ and GWB had access to the data. KK, SMD, BJG, ASJ and GWB were involved in manuscript revision, intellectual revisions and mentorship. All authors read and approved the final manuscript.

\section{Funding}

Dr. Saraschandra Vallabhajosyula is supported by the Clinical and Translational Science Award (CTSA) Grant Number UL1 TR000135 from the National Center for Advancing Translational Sciences (NCATS), a component of the National Institutes of Health $(\mathrm{NIH})$. Its contents are solely the responsibility of the authors and do not necessarily represent the official view of $\mathrm{NIH}$. This research did not receive any specific grant from funding agencies in the public, commercial or not-for-profit sectors.

\section{Availability of data and materials}

The datasets used and/or analyzed during the current study are publicly available with the Agency for Healthcare Research and Quality.

\section{Ethics approval and consent to participate}

Exempted due to the publicly available de-identified data.

\section{Consent for publication}

Not applicable.

\section{Competing interests}

Dr. Jaffe has been a consultant for Beckman, Abbott, Siemens, ET Healthcare, Sphingotec, Quidel, Brava and Novartis. All other authors have reported that they have no relationships relevant to the contents of this paper to disclose.

\begin{abstract}
Author details
${ }^{1}$ Department of Cardiovascular Medicine, Mayo Clinic, 200 First Street SW, Rochester, MN 55905, USA. ${ }^{2}$ Division of Pulmonary and Critical Care Medicine, Department of Medicine, Mayo Clinic, Rochester, MN, USA. ${ }^{3}$ Division of Nephrology and Hypertension, Department of Medicine, Mayo Clinic, Rochester, MN, USA. ${ }^{4}$ Department of Health Science Research, Robert D. and Patricia E. Kern Center for the Science of Health Care Delivery, Mayo Clinic, Rochester, MN, USA. ${ }^{5}$ Division of Hospital Internal Medicine, Department of Medicine, Mayo Clinic, Rochester, MN, USA.
\end{abstract}

Received: 26 February 2019 Accepted: 20 August 2019 Published online: 28 August 2019

\section{References}

1. Goldberg RJ, Spencer FA, Gore JM, Lessard D, Yarzebski J. Thirty-year trends (1975 to 2005) in the magnitude of, management of, and hospital death rates associated with cardiogenic shock in patients with acute myocardial infarction: a population-based perspective. Circulation. 2009;119(9):1211-9.

2. Babaev A, Frederick PD, Pasta DJ, Every N, Sichrovsky T, Hochman JS. Trends in management and outcomes of patients with acute myocardial infarction complicated by cardiogenic shock. JAMA. 2005;294(4):448-54.

3. Vallabhajosyula S, O'Horo JC, Antharam P, Ananthaneni S, Vallabhajosyula S, Stulak JM, et al. Concomitant intra-aortic balloon pump use in cardiogenic shock requiring veno-arterial extracorporeal membrane oxygenation. Circ Cardiovasc Interv. 2018;11(9):e006930.

4. Vallabhajosyula S, O'Horo JC, Antharam P, Ananthaneni S, Vallabhajosyula S, Stulak JM, et al. Venoarterial extracorporeal membrane oxygenation with concomitant Impella versus venoarterial extracorporeal membrane oxygenation for cardiogenic shock. ASAIO J. 2019. https://doi. org/10.1097/MAT.0000000000001039.

5. Hochman JS, Sleeper LA, Webb JG, Sanborn TA, White HD, Talley JD, et al. Early revascularization in acute myocardial infarction complicated by cardiogenic shock. SHOCK Investigators. Should we emergently revascularize occluded coronaries for cardiogenic shock. N Engl J Med. 1999;341(9):625-34.
6. Esposito ML, Kapur NK. Acute mechanical circulatory support for cardiogenic shock: the "door to support" time. F1000Research. 2017;6:737.

7. Vallabhajosyula S, Dunlay SM, Prasad A, Kashani K, Sakhuja A, Gersh BJ, et al. Acute noncardiac organ failure in acute myocardial infarction with cardiogenic shock. J Am Coll Cardiol. 2019;73(14):1781-91.

8. van Diepen S, Katz JN, Albert NM, Henry TD, Jacobs AK, Kapur NK, et al. Contemporary management of cardiogenic shock: a scientific statement from the American Heart Association. Circulation. 2017;136(16):e232-68.

9. Vallabhajosyula S, Barsness GW, Vallabhajosyula S. Multidisciplinary teams for cardiogenic shock. Aging. 2019;11(14):4774-6.

10. Gray A, Goodacre S, Newby DE, Masson M, Sampson F, Nicholl J. Noninvasive ventilation in acute cardiogenic pulmonary edema. N Engl J Med. 2008;359(2):142-51.

11. Hongisto M, Lassus J, Tarvasmaki T, Sionis A, Tolppanen H, Lindholm MG, et al. Use of noninvasive and invasive mechanical ventilation in cardiogenic shock: a prospective multicenter study. Int J Cardiol. 2017;230:191-7.

12. Mehta AB, Syeda SN, Wiener RS, Walkey AJ. Epidemiological trends in invasive mechanical ventilation in the United States: a population-based study. J Crit Care. 2015;30(6):1217-21.

13. Sinha SS, Sjoding MW, Sukul D, Prescott HC, Iwashyna TJ, Gurm HS, et al. Changes in primary noncardiac diagnoses over time among elderly cardiac intensive care unit patients in the United States. Circ Cardiovasc Qual Outcomes. 2017;10(8):e003616.

14. Jentzer JC, Bennett C, Wiley BM, Murphree DH, Keegan MT, Gajic O, et al. Predictive value of the sequential organ failure assessment score for mortality in a contemporary cardiac intensive care unit population. J Am Heart Assoc. 2018;7(6):e008169.

15. Introduction to the HCUP Nationwide Inpatient Sample 2009. HCUP. http://www.hcup-us.ahrq.gov/db/nation/nis/NIS_2009_INTRODUCTI ON.pdf. Accessed 18 Jan 2015.

16. Jolley RJ, Quan H, Jette N, Sawka KJ, Diep L, Goliath J, et al. Validation and optimisation of an ICD-10-coded case definition for sepsis using administrative health data. BMJ Open. 2015;5(12):e009487.

17. Lauridsen MD, Gammelager H, Schmidt M, Nielsen H, Christiansen CF. Positive predictive value of International Classification of Diseases, 10th revision, diagnosis codes for cardiogenic, hypovolemic, and septic shock in the Danish National Patient Registry. BMC Med Res Methodol. 2015;15:23.

18. Coloma PM, ValkhoffVE, Mazzaglia G, Nielsson MS, Pedersen L, Molokhia $M$, et al. Identification of acute myocardial infarction from electronic healthcare records using different disease coding systems: a validation study in three European countries. BMJ Open. 2013;3(6):e002862.

19. Lambert L, Blais C, Hamel D, Brown K, Rinfret S, Cartier R, et al. Evaluation of care and surveillance of cardiovascular disease: can we trust medicoadministrative hospital data? Can J Cardiol. 2012;28(2):162-8.

20. Gupta S, Sakhuja A, Kumar G, McGrath E, Nanchal RS, Kashani KB. Culture-negative severe sepsis: nationwide trends and outcomes. Chest. 2016;150(6):1251-9.

21. Kumar G, Kumar N, Taneja A, Kaleekal T, Tarima S, McGinley E, et al. Nationwide trends of severe sepsis in the 21st century (2000-2007). Chest. 2011;140(5):1223-31.

22. Vallabhajosyula S, Dunlay SM, Kashani K, Vallabhajosyula S, Vallabhajosyula S, Sundaragiri PR, et al. Temporal trends and outcomes of prolonged invasive mechanical ventilation and tracheostomy use in acute myocardial infarction with cardiogenic shock in the United States. Int J Cardiol. 2019;285:6-10.

23. Walkey AJ, Lagu T, Lindenauer PK. Trends in sepsis and infection sources in the United States. A population-based study. Ann Am Thorac Soc. 2015;12(2):216-20.

24. Vallabhajosyula S, Arora S, Lahewala S, Kumar V, Shantha GPS, Jentzer JC, et al. Temporary mechanical circulatory support for refractory cardiogenic shock before left ventricular assist device surgery. J Am Heart Assoc. 2018;7(22):e010193.

25. Vallabhajosyula S, Arora S, Sakhuja A, Lahewala S, Kumar V, Shantha GPS, et al. Trends, predictors, and outcomes of temporary mechanical circulatory support for postcardiac surgery cardiogenic shock. Am J Cardiol. 2019;123(3):489-97.

26. Vallabhajosyula S, Dunlay SM, Barsness GW, Rihal CS, Holmes DR Jr, Prasad A. Hospital-level disparities in the outcomes of acute myocardial infarction with cardiogenic shock. Am J Cardiol. 2019;124(4):491-8. 
27. Vallabhajosyula S, Dunlay SM, Murphree DH, Barsness GW, Sandhu GS, Lerman A, et al. Cardiogenic shock in Takotsubo cardiomyopathy versus acute myocardial infarction: an 8-year national perspective on clinical characteristics, management, and outcomes. JACC Heart Fail. 2019;7(6):469-76.

28. Vallabhajosyula S, Prasad A, Dunlay SM, Murphree DH Jr, Ingram C, Mueller PS, et al. Utilization of palliative care for cardiogenic shock complicating acute myocardial infarction: a 15-year national perspective on trends, disparities, predictors, and outcomes. J Am Heart Assoc. 2019;8(15):e011954

29. Vallabhajosyula S, Ya'Qoub L, Dunlay SM, Vallabhajosyula S, Vallabhajosyula S, Sundaragiri PR, et al. Sex disparities in acute kidney injury complicating acute myocardial infarction with cardiogenic shock. ESC Heart Fail. 2019;6(4):874-7.

30. Deyo RA, Cherkin DC, Ciol MA. Adapting a clinical comorbidity index for use with ICD-9-CM administrative databases. J Clin Epidemiol. 1992;45(6):613-9.

31. Khera R, Krumholz HM. With great power comes great responsibility: big data research from the National Inpatient Sample. Circ Cardiovasc Qual Outcomes. 2017;10(7):e003846.

32. Thiele H, Zeymer U, Neumann FJ, Ferenc M, Olbrich HG, Hausleiter J, et al. Intraaortic balloon support for myocardial infarction with cardiogenic shock. N Engl J Med. 2012;367(14):1287-96.

33. Vallabhajosyula S, Kumar M, Pandompatam G, Sakhuja A, Kashyap R, Kashani K, et al. Prognostic impact of isolated right ventricular dysfunction in sepsis and septic shock: an 8-year historical cohort study. Ann Intensive Care. 2017;7(1):94.
34. Pinsky MR. Heart lung interactions during mechanical ventilation. Curr Opin Crit Care. 2012;18(3):256-60.

35. Jentzer JC, Vallabhajosyula S, Khanna AK, Chawla LS, Busse LW, Kashani KB. Management of refractory vasodilatory shock. Chest. 2018;154:416-26

36. Morrow DA, Fang JC, Fintel DJ, Granger CB, Katz JN, Kushner FG, et al. Evolution of critical care cardiology: transformation of the cardiovascular intensive care unit and the emerging need for new medical staffing and training models: a scientific statement from the American Heart Association. Circulation. 2012;126(11):1408-28.

37. Sakr Y, Lobo SM, Moreno RP, Gerlach H, Ranieri VM, Michalopoulos A, et al. Patterns and early evolution of organ failure in the intensive care unit and their relation to outcome. Crit Care. 2012;16(6):R222.

38. Poss J, Koster J, Fuernau G, Eitel I, de Waha S, Ouarrak T, et al. Risk stratification for patients in cardiogenic shock after acute myocardial infarction. J Am Coll Cardiol. 2017;69(15):1913-20.

39. Harjola VP, Lassus J, Sionis A, Kober L, Tarvasmaki T, Spinar J, et al. Clinical picture and risk prediction of short-term mortality in cardiogenic shock. Eur J Heart Fail. 2015;17(5):501-9.

40. Spinar J, Parenica J, Vitovec J, Widimsky P, Linhart A, Fedorco M, et al. Baseline characteristics and hospital mortality in the Acute Heart Failure Database (AHEAD) Main registry. Crit Care. 2011;15(6):R291.

\section{Publisher's Note}

Springer Nature remains neutral with regard to jurisdictional claims in published maps and institutional affiliations.

\section{Submit your manuscript to a SpringerOpen ${ }^{\circ}$ journal and benefit from:}

- Convenient online submission

- Rigorous peer review

- Open access: articles freely available online

- High visibility within the field

- Retaining the copyright to your article

Submit your next manuscript at $\boldsymbol{\nabla}$ springeropen.com 